Electronic Journal of Research in Educational Psychology

\title{
Revisores 2020
}


Abellán Roselló, Laura. Universidad Jaume I de Castellón.

Adi Nurcahyo, Firmanto.Universitas Gadjah Mada, Yogyakarta.

Álvarez Fernández, Lourdes. Universidad de León.

Angulo Brunet, Adriana. Universidad Autónoma de Barcelona.

Arias Gundín, Olga. Universidad de León.

Armelim Almiro, Pedro. University of Lisbon Luis de Camoes (Portugal)

Axpe Sáez, Inge. Universidad del País Vasco.

Baña Castro, Manoel. Universidad de la Coruña.

Bautista García, Victoria Eugenia. Universidad de Málaga

Blanco Fernández, Jana. Universidad de León.

Boque Martorell, María Carme. Universidad Ramón Llull.

Calero, Alejandra Daniela. Universidad de Buenos Aires

Cantero García, María. Universidad Autónoma de Madrid.

Gabari Gambarte, María Ines. Universidad Pública de Navarra.

Carbonero Martín, Miguel Angel. Universidad de Valladolid.

García Gómez, Andres. Universidad de Extremadura.

Caurcel Cara, María Jesús. Universidad de Granada.

Cabedo Mas, Alberto. Universitat Jaume I de Castellón.

Campayo Muñoz, María Angeles. Universidad Complutense de Madrid.

Castilla Mesa, Teresa. Universidad de Málaga.

Colledani, Daiana, Universidad de Padova (Italia).

Domenech Betoret, Fernando. Universidad Jaume I de Castellón.

Fadda, Salvatore. Universidad de Sassari (Italia).

Fernández Baena, Francisco Javier. Universidad de Málaga.

Férnandez Simo, Jesús Deibe. Universidad de Vigo.

Flores Lucas, María del Valle. Universidad de Valladolid.

Freire Rodríguez, Carlos. Universidad de la Coruña.

Gil Flores, Javier. Universidad de Sevilla.

Gimeno Collado, Adelina. Universidad de Valencia.

González García, Carla. Universidad de Oviedo.

Gómez Baya, Diego. Universidad de Huelva.

Inglés Saura, Cándido José. Universidad Miguel Hernández de Elche.

Jiménez García, Lucía. Universidad de Sevilla.

Lago, José Ramón, Universidad de Vic. 
Lázaro Visa, Susana. Universidad de Cantabria.

Luque Vilaseca, Juan Luis. Universidad de Málaga.

Maldonado Rico, Antonio. Universidad Autónoma de Madrid.

Martí Vilar, Manuel. Universidad de Valencia.

Martín Palacio, María Eugenia. Universidad Complutense de Madrid.

Martínez Fernández, José Reinaldo. Universitad Autónoma de Barcelona.

Martínez Ramón, Juan Pedro. Universidad de Murcia.

Medina Gómez, Begona. Universidad de Burgos.

Méndez Carrillo, Francisco Javier. Universidad de Murcia.

Méndez Mateo, María Inmaculada. Universidad de Murcia.

Moé Angélica. Universitá Degli Studi di Padova.

Moreno Bataller, Cecilia Beatriz. Universidad Católica Argentina (Mendoza).

Moriana Elvira, Juan Antonio. Universidad de Córdoba.

Muñoz Quezada, María Teresa. Universidad Católica del Maule.

Navarro Guzmán, José Ignacio. Universidad de Cádiz.

Navas Martínez, Leandro. Universidad de Alicante.

Naya Riveriro, María Cristina. Universidad de la Coruña

Nortes Martínez-Artero, Rosa. Universidad de Murcia.

Ojeda Pérez, Mónica. Universidad de Sevilla.

Ortega Rivera, Francisco Javier. Universidad de Sevilla.

Pinto Marín, Edel. Universidad del Desarrollo (Chile).

Polo Sánchez, Tamara. Universidad de Granada.

Pozo Municio, Juan Ignacio. Universidad Autónoma de Madrid.

Pozo Rico, Teresa. Universidad de Alicante.

Prieto Ursúa, Universidad Pontificia de Comillas.

Puertas González, José Antonio. Universidad de Granada.

Rivas García, Sandra María. Universidad de Granada.

Rodríguez de Dios, Isabel. Universidad Loyola de Sevilla.

Rodríguez Meirinhos, Ana. Universidad Loyola Andalucía.

Rodríguez Pérez, Celestino. Universidad de Oviedo.

Rodríguez Sánchez, Manuel, Universidad Complutense de Madrid

Rojas Tejada, Antonio. Universidad de Almería.

Sala Roca, Josefina. Universitat Autónoma de Barcelona.

Saldaña Sage, David. Universidad de Sevilla. 
Sánchez Hernández, Óscar. Universidad de Murcia.

Sánchez Peralta, Francisco Javier. Universidad de Almería.

Samper García, Paula. Universidad de Valencia.

Sastre Llorente, Santiago. Universidad Complutense de Madrid.

Tárraga Mínguez, Raúl. Universidad de Valencia.

Tourón, Javier. Universidad Internacional de la Rioja (UNIR).

Valadez Sierra, María de los Dolores. Universidad de Guadalajara.

Valero García, Ana Vanesa. Universidad de Murcia.

Vicente de Vera, María Inmaculada. Universidad Pública de Navarra.

Vieiro iglesias, Pilar. Universidad de la Coruña.

Vigo Arrazola, María Begoña. Universidad de Zaragoza.

Vizoso Gómez, Carmen María. Universidad de León.

Recibidos: 137 artículos

Rechazados: 70 artículos

Aceptados: 30 artículos

Tasa de aceptación: 21,9\%

Tasa de rechazo: $78,1 \%$ 